\title{
Assessment of individual renal function in children using ${ }^{99 \mathrm{~m} T c-D T P A}$
}

\author{
G. KAINER, B. MCILVEEN, R. HÖSCHL, AND A. R. ROSENBERG \\ Department of Nuclear Medicine, and Division of Nephrology, Prince of Wales Children's Hospital, \\ New South Wales
}

SUMMARY Differential renal function assessment using ${ }^{99 m}$ Tc-DTPA was carried out in 12 children aged between 3 weeks and 11 years who had undergone surgical procedures which allowed separate access to the urine output from each kidney. The results correlated well with those obtained by measuring individual kidney creatinine clearances. The best result was obtained using the technique of deconvolution analysis $(\mathrm{r}=0.98 ; \mathrm{P}<0.001)$. We conclude that the method is accurate and recommend its use in the management and follow-up of patients with asymmetric renal disease.

The need for a reliable noninvasive method for studying individual kidney function in children is apparent. The damage incurred by the kidneys in diseases such as vesicoureteric reflux and obstructive uropathy is seldom symmetrical, and the selection of optimal treatment and follow-up requires quantitative information about the functional status of each kidney as well as knowledge of the total renal function. An example of the type of case in which such information would be useful is that of a child with bilateral reflux nephropathy who has poor function of one kidney on intravenous urography. A decision has to be made as to whether ureteric reimplantation or nephrectomy should be carried out. It would obviously be advantageous if the relative function of the affected kidney could accurately be assessed.

Radiographic contrast techniques give valuable information about the anatomical structure of the kidneys and collecting systems. However, they provide only qualitative information on differential renal function. Bilateral ureteric cannulation for the measurement of individual kidney clearances carries the risk of introducing infection and requires general anaesthesia, and is therefore not suitable for routine use.

Attempts have been made for some time to assess individual renal function by noninvasive radioisotope

\footnotetext{
Prince of Wales Children's Hospital, New South Wales, Australia

A. R. ROSENBERG, paediatric nephrologist

G. KAINER, paediatric registrar

B. MCILVEEN, senior physicist

R. HösCHL, physician in nuclear medicine
}

techniques (Johnston and Irving, 1967; Kathel, 1971), and more recently methods using technetiumlabelled stannous diethylenetriaminepentaacetic acid ( ${ }^{99 m}$ Tc-DTPA) have been described (Pieretti et al., 1974; Nielsen et al., 1977). The use of deconvolution analysis to quantify the renogram and the potential advantages of this method in radioisotopic assessment of renal function have been described by Kenny et al. (1975) and Diffey et al. (1976).

A method for quantitating individual renal function using ${ }^{89 m}$ Tc-DTPA has recently been described by Piepsz et al. (1977), but nonradioisotopic measurements of individual renal function were not carried out and it is therefore difficult to judge the accuracy of the technique.

The purpose of this study was to determine the accuracy of individual renal function measurements in children using ${ }^{99 m}$ Tc-DTPA.

\section{Methods and materials}

The 12 children studied were aged between 3 weeks and 11 years (Table 1). Informed parental consent was obtained in each case. All had undergone surgical procedures which allowed separate access to the urine output from each of their kidneys-that is, they had ureterostomies or indwelling ureteric splints. Renal function assessments were carried out at least 6 days after surgery.

Urine was collected from both kidneys simultaneously for a period of not less than 6 hours, and plasma creatinine was estimated on a blood sample taken at the time of the 'urine collection. All creatinine estimations were done using the auto- 
Table 1 Results of individual kidney function assessment in 12 children

\begin{tabular}{|c|c|c|c|c|c|c|c|c|c|c|c|c|}
\hline \multirow[t]{3}{*}{ Case } & \multirow[t]{3}{*}{ Age } & \multirow[t]{3}{*}{ Diagnosis } & \multirow{2}{*}{\multicolumn{2}{|c|}{$\begin{array}{l}\% \text { creatinine } \\
\text { clearance }\end{array}$}} & \multicolumn{8}{|c|}{$\%$ uptake } \\
\hline & & & & & \multicolumn{2}{|c|}{$1-3 \min$} & \multicolumn{2}{|c|}{$2-4 \min$} & \multicolumn{2}{|c|}{$13-15 \mathrm{~min}$} & \multicolumn{2}{|c|}{ Deconvolution } \\
\hline & & & Left & Right & Left & Right & Left & Right & Left & Right & Left & Right \\
\hline 1 & $6 \frac{1}{2}$ years & Prune-belly syndrome & 7 & 93 & 12 & 88 & 13 & 87 & 3 & 97 & 13 & 87 \\
\hline 2 & $10 \frac{1}{2}$ years & Bilateral reflux & 21 & 79 & 20 & 80 & 21 & 79 & 10 & 90 & 25 & 75 \\
\hline 3 & 17 months & Bilateral reflux & 56 & 44 & 53 & 47 & 54 & 46 & 47 & 53 & 55 & 45 \\
\hline 4 & $2 t$ years & Bilateral reflux & 33 & 67 & 26 & 74 & 23 & 77 & 18 & 82 & 24 & 76 \\
\hline 5 & 14 months & Bilateral reflux & 83 & 17 & 66 & 34 & 69 & 31 & 44 & 56 & 76 & 24 \\
\hline 6 & $6 \frac{1}{2}$ years & R. renal agenesis & 100 & 0 & 93 & 7 & 96 & 4 & 97 & 3 & 100 & 0 \\
\hline 7 & 19 months & Bilateral reflux & 40 & 60 & 44 & 56 & 44 & 56 & 42 & 58 & 48 & 52 \\
\hline 8 & 7 months & Bilateral reflux & 55 & 45 & 51 & 49 & 50 & 50 & 46 & 54 & 51 & 49 \\
\hline 9 & 8 months & $\begin{array}{l}\text { L. pelviureteric junction } \\
\text { obstruction }\end{array}$ & 38 & 62 & 32 & 68 & 32 & 68 & 56 & 44 & 38 & 62 \\
\hline 10 & 3 weeks & Bilateral hydronephrosis & 23 & 77 & 19 & 81 & 22 & 78 & 29 & 71 & 24 & 76 \\
\hline 11 & 3 weeks & Bilateral hydronephrosis & 25 & 75 & 27 & 73 & 26 & 74 & 23 & 77 & 24 & 76 \\
\hline 12 & 3 weeks & Bilateral hydronephrosis & 37 & 63 & 26 & 74 & 27 & 73 & 26 & 74 & 43 & 57 \\
\hline
\end{tabular}

analyser (6-60 Technicon). To establish the stability of the patient's renal status, two plasma creatinine estimations a day apart were performed. Individual kidney creatinine clearances were derived using the standard formula clearance $=U . V / P$ (where $U=$ concentration of creatinine in urine, $\mathrm{V}=$ volume of urine collected per minute, $P=$ plasma creatinine concentration). The contribution of the left kidney was expressed as a percentage of the sum of the creatinine clearances of the two kidneys.

No limitations on food and drink were imposed during the estimation of creatinine clearance or before scanning, and none of the patients was receiving intravenous fluids. Scans were performed within 24 hours of creatinine clearance estimations. All the children were recumbent for at least one hour before scanning. Premedication was not routinely given beforehand, but some children required a small dose of diazepam immediately before injection of the radiopharmaceutical. To allay anxiety the parents were asked to be present during the test.

An intravenous dose of 3.7 megabecquerels $(100 \mu \mathrm{Ci})$ per $\mathrm{kg}$ of ${ }^{99 \mathrm{~m} T c-D T P A}$ (supplied by the Australian Atomic Energy Commission) was injected as a bolus. The children lay on a foam rubber pad placed directly on the gamma camera head (Nuclear Chicago Pho-Gamma III HP Camera with low energy, high sensitivity parallel hole collimator). Data were recorded by a computer (DEC Gamma-11 system) at the rate of one frame per 5 seconds for a total of 200 frames.

Analogue scans were recorded on Polaroid and $70 \mathrm{~mm}$ films for one minute after the injection (one exposure every 5 seconds), followed by renal scan image (taken at a preset count of 300000 ). For computer processing, regions of interest were drawn manually as close as possible to the renal outlines using a 'joystick'. In each subnephric area a back- ground region of interest was chosen, care being taken to avoid the course of the ureters and large vessels. Another region was selected over the heart in order to generate the blood activity (heart) curve (Figs 1 and 2).

Counts for each kidney were corrected for background after the background area was normalised to the area of the ipsilateral kidney. The 120 -second net counts for each kidney were integrated over the time intervals 1-3 minutes, 2-4 minutes, and 13-15 minutes. Background corrected time activity curves were displayed (Fig. 3).

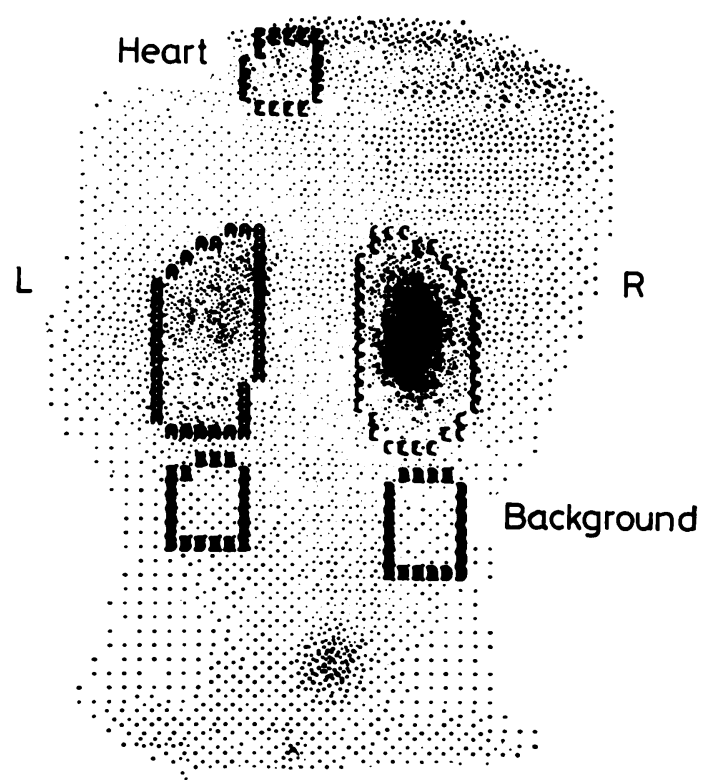

Fig. 1 Case 9. Gamma camera image. Regions of interest drawn around the kidneys, heart, and background areas. 


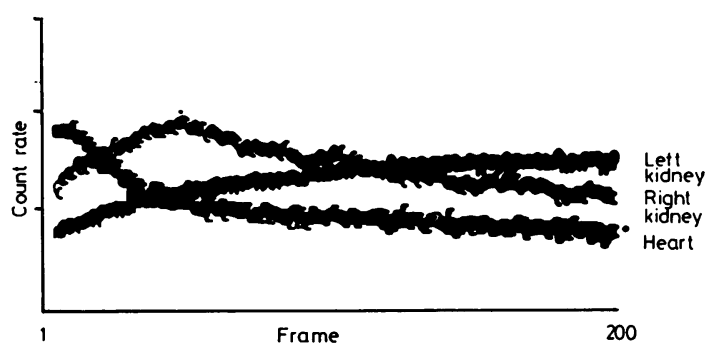

Fig. 2 Case 9. Renograms and heart curve. Right kidney curve normal. Left kidney curve shows impaired renal function.

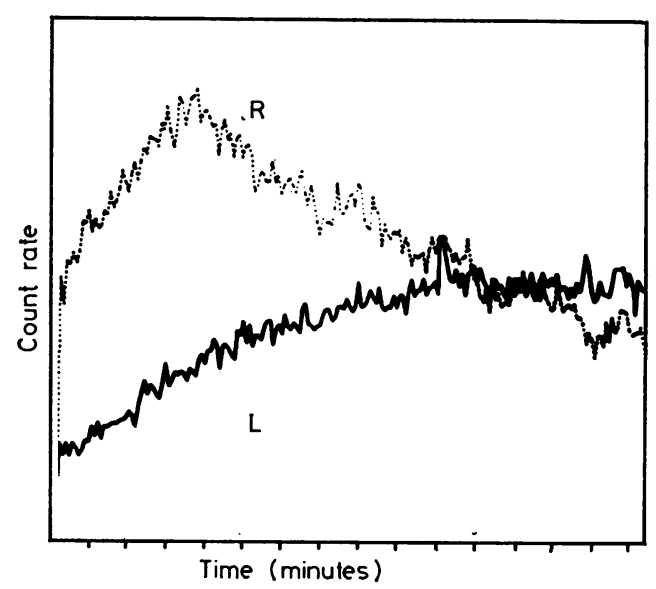

Fig. 3 Case 9. Renograms after background subtraction. Right $(R)$ kidney curve normal. Left $(L)$ kidney curve shows impaired renal function.

The percentage uptake of radioactivity for the left kidney was calculated by dividing the net counts for that kidney by the sum of the net counts from both kidneys - that is $\mathbf{L} /(\mathbf{L}+\mathbf{R}) \times 100$. The results for the three time intervals were compared with the percentage contribution of the left kidney to the total creatinine clearance (Figs 5a, 5b, 5c).

Deconvolution analysis with data bounding (Diffey and Corfield, 1975; Valentinuzzi and Montaldo Volachec, 1975) was used to plot the retention function for each of the kidneys. The retention function is the form that the renogram would take if a bolus of ${ }^{99 \mathrm{~m} T c-D T P A}$ could be injected directly into the renal artery (Fig. 4). The amplitudes of the retention function curves were used to calculate the relative uptake of radioactivity by the left kidney as compared with the total, and the results compared with those obtained from creatinine clearances (Fig. 5d).

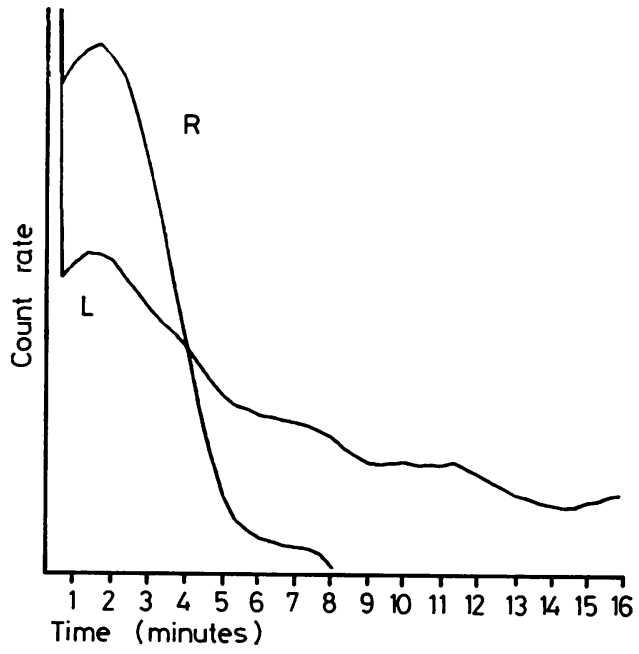

Fig. 4 Case 9. Retention function curves derived from deconvolution analysis with data bounding. Right $(R)$ kidney curve normal. Left $(L)$ kidney curve shows impaired renal function.

Correlation coefficients were derived by comparing the results obtained from scanning with those obtained from creatinine clearance estimations (Fig. 5).

$95 \%$ confidence limits were determined for the results obtained from scanning methods at 1-3 minutes, 2-4 minutes, and by deconvolution analysis. Two points were chosen for determination of confidence limits-namely, where the left kidney contribution to the total renal function was 10 and $90 \%$ (Table 2).

\section{Results}

The data are presented in Table 1. The contribution of the left kidney to the total creatinine clearance,

Table 2 Accuracy of scanning methods for predicting individual kidney function at 10 and $90 \%$ of overall renal function

\begin{tabular}{ll}
\hline $\begin{array}{l}\text { Left kidney \% uptake from } \\
\text { scans }\end{array}$ & $\begin{array}{l}\text { Prediction for \% clearance of } \\
\text { left kidney with } 95 \% \text { confidence } \\
\text { limits }\end{array}$ \\
\hline $1-3$ min & $10 \cdot 8 \pm 6 \cdot 1$ \\
10 & $99.9 \pm 11.0$ \\
90 & $11.0 \pm 6 \cdot 2$ \\
$2-4$ min & $97.4 \pm 9.1$ \\
10 & $8 \cdot 2 \pm 5 \cdot 9$ \\
90 & $91.9 \pm 7.5$ \\
Deconvolution & \\
10 & \\
90 & \\
\hline
\end{tabular}

*Regression analysis. 

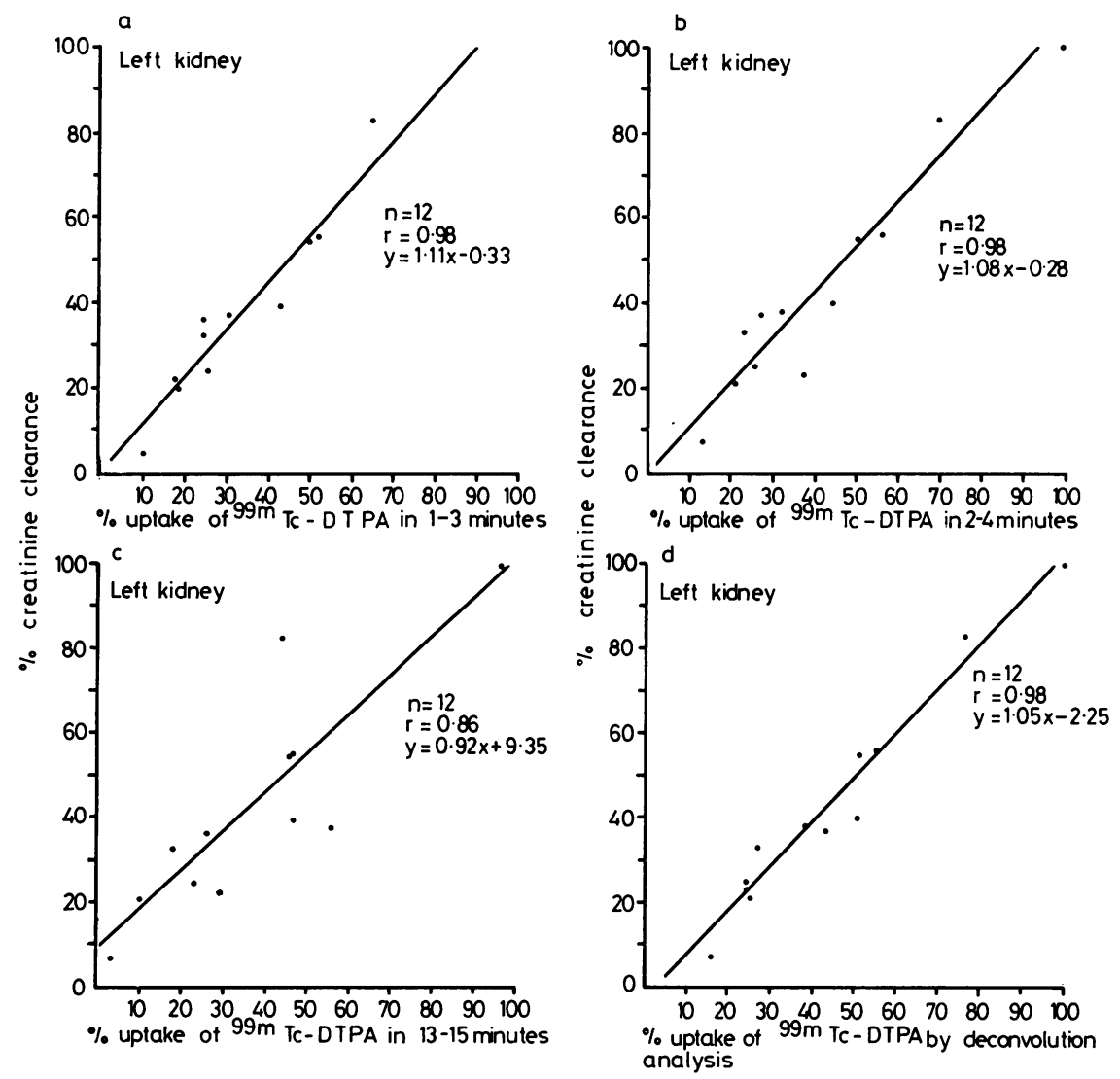

Fig. 5 Left kidney. Percentage of total creatinine clearance $v$. percentage of total renal uptake of ${ }^{99 \mathrm{~m} T c-D T P A}(a)$ in $1-3 \min ,(b)$ in 2-4 min, (c) in 13-15 min, (d) by deconvolution analysis.

when compared with the results obtained using radioisotopic techniques, showed a high degree of correlation $(r=0.98)$ with the 1-3 minute and 2-4 minute kidney uptake ratios and with the deconvolution analysis technique. A lower correlation $(r=0.86)$ was found at the 13-15 minute interval. All were significant $(P<0 \cdot 001)$.

The mean disparity between the scan results and those obtained from creatinine clearances was $6 \%$ for the $1-3$ minute, $5 \%$ for the $2-4$ minute, and $11 \%$ for the 13-15 minute uptake ratios, and $4 \%$ for the deconvolution analysis method.

The calculated predictions for left renal function from the data, using regression analysis, are presented in Table 2.

\section{Discussion}

The required characteristics of any substance for the measurement of glomerular filtration rate (GFR) are: (1) It must be preferentially excreted by the kidney. (2) It must be freely filtered through the glomerular basement membrane. (3) It must be physiologically inert. (4) It must not be reabsorbed or secreted by the renal tubules.

The classical substance fulfilling the above criteria is inulin (Materson, 1971). However, determination of inulin concentration is difficult and the technique does not lend itself to routine hospital use. Inulin is not readily labelled by available gamma-emitting radionuclides of suitable energy, radiation, or half-life. One of the newly developed substances with physiological characteristics closely resembling those of inulin is DTPA (Reba et al., 1974; Barbour et al., 1976). This chelating agent can be readily labelled with various radionuclides, including ${ }^{99 \mathrm{~m}} \mathrm{Tc}$. The resulting complex is suitable for radioisotopic determination of GFR (Hauser et al., 1970; Chevru et al., 1974).

${ }^{99 m}$ Tc has ideal physical characteristics for application in nuclear medicine. It has a short half-life (6 hours) and suitable radiation characteristics ( $140 \mathrm{keV}$ photons in $90 \%$ abundance and no beta radiation). The $140 \mathrm{keV}$ photon has adequate tissue 
penetration and is efficiently measured by the current generation of gamma cameras. Its low radiation dose permits repeated examinations in children (Chevru et al., 1974; Kirchner et al., 1975).

The ${ }^{99 m}$ Tc-DTPA complex, after intravenous injection, equilibrates rapidly throughout its extracellular distribution space. It is cleared through the glomeruli without appreciable tubular secretion or reabsorption and only $5 \%$ is excreted in the faeces. More than $98 \%$ of the radioactivity remains in the chelated form and only $5-10 \%$ of the serum radioactivity at one hour after injection is associated with serum proteins (Hauser et al., 1970; Arnold et al., 1975).

This study demonstrated that individual renal function in children can accurately be assessed using ${ }^{99 m}$ Tc-DTPA and external monitoring with computerised quantitation. The deconvolution analysis method gave the best results, but the 1-3 minute and 2-4 minute kidney uptake ratios were also satisfactory.

Statistical testing of the accuracy of these methods at the extremes of renal function-that is 10 and $90 \%$ function in one kidney, gave results quite adequate for clinical use (Table 2). The best methods for measuring GFR have an accuracy of $\pm 3-6 \%$ (Materson, 1971). 10 and $90 \%$ levels of individual renal function were chosen because it is at these levels of relative function that decisions regarding nephrectomy are most often made.

On computer analysis of the gamma camera data, it is essential to draw the regions of interest as closely as possible around the renal outlines; this is particularly important for the relative uptake method. Inclusion of extrarenal areas tends to reduce the average count in each matrix element. For the deconvolution analysis method the selection of a region of interest close to the kidney outline is not as critical, provided it includes all functioning renal parenchyma.

All the children were recumbent for at least one hour before and during the time of scanning. This was felt to be important as changes in posture may lead to alterations in urodynamics and haemodynamics. The positioning of the child on top of the collimator made venepunctures easier and was found to be less frightening for the child than prone with the gamma camera head suspended above.

It was found that background regions of interest were most representative in the subnephric regions. Other sites such as areas lateral to the kidneys were found to be technically unsatisfactory in smaller children with hydronephrosis. In one child (Case 6, Table 1) with unilateral renal agenesis, the counts obtained in the subnephric area virtually equalled the counts obtained from the site where the kidney would normally have been situated.

Apart from its use in determination of individual

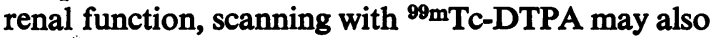
be helpful in the demonstration of anatomical abnormalities of the kidneys and collecting systems, and in the diagnosis of urinary obstruction. Obstruction to urine outflow can be inferred from the shape of the renogram and from the retention function curve after deconvolution analysis (left kidney; Figs 3 and 4). Retention of radioactive material due to obstruction is best assessed by the net cumulative count at the 13-15 minute time interval (for example, Case 8, Table 1).

In summary, we have shown that assessment of individual renal function with ${ }^{99} \mathrm{~m}$ Tc-DTPA in children is accurate. It carries less risk and is no more disturbing to the child than a comparable radiological investigation. It can be done in any hospital with a modern nuclear medicine department. We believe that the use of this technique will prove to be important in the management and follow-up of patients with asymmetric renal diseases and also in the understanding of the evolution of these disorders.

We thank Professor G. F. Murnaghan and Dr R. H. Farnsworth for allowing access to patients, and Dr G. Southon for computer programming assistance.

\section{References}

Arnold, R. W., Subramanian, G., McAfee, J. G., Blair, R. J., and Thomas, F. D. (1975). Comparison of ${ }^{90 \mathrm{~m} T c}$ complexes for renal imaging. Journal of Nuclear Medicine, 16, 357-367.

Barbour, G. L., Crumb, C. K., Boyd, C. M., Reeves, R. D., Rastogi, S. P., and Patterson, R. M. (1976). Comparison of inulin, iothalamate, and ${ }^{9} \mathrm{mTC}$-DTPA for measurement of glomerular filtration rate. Journal of Nuclear Medicine, 17, 317-320.

Chevru, L. R., Freeman, L. M., and Blaufox, M. D. (1974). Radiopharmaceuticals for renal studies. Seminars in Nuclear Medicine, 4, 3-22.

Diffey, B. L., and Corfield, J. R. (1975). Data-bounding technique in discrete deconvolution. Medical and Biological Engineering, 14, 478.

Diffey, B. L., Hall, F. M., and Corfield, J. R. (1976). The ${ }^{90} \mathrm{mTc}$-DTPA dynamic renal scan with deconvolution analysis. Journal of Nuclear Medicine, 17, 352-355.

Hauser, W., Atkins, H. L., Nelson, K. G., and Richards, P. (1970). Technetium-99mDTPA: a new radiopharmaceutical for brain and kidney scanning. Radiology, 94, 679-684.

Johnston, J. H., and Irving, I. M. (1967). Experiences with radioisotope renography in children. Archives of Disease in Childhood, 42, 583-591.

Kathel, B. L. (1971). Radioisotope renography as a renal function test in the newborn. Archives of Disease in Childhood, 46, 314-320.

Kenny, R. W., Ackery, D. M., Fleming, J. S., Goddard, B.A., and Grant, R. W. (1975). Deconvolution analysis of the scintillation camera renogram. British Journal of Radiology, 48, 481-486. 
Kirchner, P. T., James, A. E., Jr, Reba, R. C., and Wagner, H. N. Jr (1975). Patterns of excretion of radioactive chelates in obstructive uropathy. Radiology, 114, 655-661. Materson, B. J. (1971). Measurement of glomerular filtration rate. CRC Critical Reviews in Clinical Laboratory Sciences, 2, 1-43.

Nielsen, S. P., Møller, M. L., and Trap-Jensen, J. (1977). ${ }^{90 \mathrm{~m} T c-D T P A}$ scintillation-camera renography: a new method for estimation of single-kidney function. Journal of Nuclear Medicine, 18, 112-117.

Piepsz, A., Dobbeleir, A., and Erbsmann, F. (1977). Measurement of separate kidney clearance by means of $99 \mathrm{~m}$-TcDTPA complex and a scintillation camera. European Journal of Nuclear Medicine, 2, 173-177.

Pieretti, R., Gilday, D., and Jeffs, R. (1974). Differential kidney scan in paediatric urology. Urology, 4, 665-668.
Reba, R. C., Poulose, K. P., and Kirchner, P. T. (1974). Radiolabelled chelates for visualization of kidney function and structure with emphasis on their use in renal insufficiency. Seminars in Nuclear Medicine, 4, 151-168.

Valentinuzzi, M. E., and Montaldo Volachec, M. E. (1975). Discrete deconvolution. Medical and Biological Engineering, 13, 123-125.

Correspondence to Dr A. R. Rosenberg, Prince of Wales Children's Hospital, Randwick, New South Wales 2031, Australia.

Received 16 January 1979 